\title{
A prática grupal e o discurso sobre a ética em documentos oficiais
}

\author{
Group work and the discourse of \\ ethics in official documents
}

\author{
Emerson Fernando RASERA ${ }^{1}$ \\ Flavia Miranda OLIVEIRA ${ }^{1}$ \\ Mario José Bertini Silva de JESUS
}

\begin{abstract}
Resumo
Os profissionais envolvidos na prática grupal enfrentam desafios éticos característicos do contexto grupal. Buscando contribuir para a reflexão sobre o discurso ético nas práticas grupais, esse artigo tem por objetivo compreender de que forma as normatizações éticas de organizações voltadas à prática grupal descrevem e propõem a ética em grupo. Metodologicamente, realizamos uma análise crítica dos principais temas presentes nos documentos da American Group Psychotherapy Association, International Association for Group Psychotherapy and Group Processes e Association of Specialists in Group Work. A análise identificou: a) princípios de bioética principialista em todos os documentos; b) diferentes descrições de grupo, coordenador e participante; e c) ênfase ética no terapeuta como profissional, mais do que na prática grupal. A análise realizada sugere que os documentos buscam contribuir com a institucionalização da profissão e respondem a uma demanda social por responsabilização pela prática profissional.
\end{abstract}

Palavras-chave: Códigos de ética; Dinâmica de grupo; Psicoterapia de grupo.

\begin{abstract}
Professionals involved with group work face ethical challenges specific to the group context. Seeking to contribute to an ethical discourse in group work, this article aims to understand how the ethical norms of group work organizations define group ethics. Methodologically, we conducted a critical analysis of the major themes found in ethical documents of the American Group Psychotherapy Association, the International Association for Group Psychotherapy and Group Processes, and the Association of Specialists in Group Work. The analysis identified: a) principles of bioethics principlism in all documents, b) different descriptions of group work, the group leader and the participant, and c) an emphasis on ethics for the therapist as a professional, rather than for group work. The analysis suggests that the documents seek to contribute to the institutionalization of the profession and meet a social demand for accountability of the professional practice.
\end{abstract}

Keywords: Codes of ethics; Group dynamics; Psychotherapy group.

$\nabla \nabla v$

1 Universidade Federal de Uberlândia, Instituto de Psicologia, Programa de Pós-Graduação em Psicologia. Campus Umuarama, Av. Pará, 1720, Bloco 2C, Sala 2C47, 38405-320, Uberlândia, MG, Brasil. Correspondência para/Correspondence to: E.F. RASERA. E-mail: <emersonrasera@gmail.com>.

Apoio: Conselho Nacional de Desenvolvimento Científico e Tecnológico (n 306316/2009-9); Coordenação de Aperfeiçoamento de Pessoal de Nível Superior. 
As questões éticas estão presentes em inúmeras atividades que realizamos em nossas vidas, com diferentes pessoas envolvidas. O contexto profissional é um dos quais, claramente, as questões éticas estão no foco da atenção, havendo, muitas vezes, a formalização de um código de ética a ser seguido na relação com os usuários dos serviços e outros profissionais.

O Conselho Federal de Psicologia (CFP), buscando atualizar e amadurecer o Código de Ética da Psicologia, já produziu três versões desse documento: em 1979, 1987 e 2005. Essas transformações buscaram contemplar outras esferas de atuação profissional, para além da clínica privada, e uma sensibilidade aos direitos coletivos. Da mesma forma, o Código teve seu caráter prescritivo redimensionado, promovendo uma reflexão sobre os conceitos de ética de forma mais ampla (Conselho Regional de Psicologia de São Paulo, 2005).

Os psicólogos brasileiros que trabalham com grupos devem seguir os preceitos éticos estabelecidos pelo Código de Ética Profissional do Psicólogo (CFP, 2005). Contudo, há algumas situações típicas do contexto grupal que demandam orientações específicas - relativas à confidencialidade, às relações entre terapeutas e membros do grupo, questões técnicas, entre outras -, àqueles que se utilizam dos dispositivos grupais em sua atuação profissional.

Na literatura, vemos como os terapeutas e os participantes de grupo enfrentam diferentes impasses éticos. Em relação à confidencialidade, os terapeutas têm dificuldades em antecipar aos participantes os limites da confidencialidade (Roback, Ochoa, Bloch, \& Purdon, 1992); os participantes, ao preservarem o sigilo sobre informações de outro participante no próprio grupo, podem acabar por limitar suas formas de participação na terapia e, consequentemente, perdem parte de seu benefício terapêutico (Hough, 1992); e a oferta de serviços oferecidos mediante os convênios de saúde dificulta a confidencialidade das informações e o acesso dos participantes a serviços apropriados (Mackenzie, 1999).

No que se refere às relações entre terapeutas 406 e participantes, há uma série de questões decorren- tes dos múltiplos papéis assumidos por terapeutas, tanto no campo das práticas de formação de terapeutas - o que faz com que muitos deles sejam ao mesmo tempo terapeutas e supervisores em relacionamentos com os mesmos pacientes e supervisonandos (Merta \& Sisson, 1991; Pepper, 2002, 2004) -, como na pesquisa, em que o terapeuta de um grupo é ao mesmo tempo pesquisador do grupo (Motta, Nunes, Munari, \& Medeiros, 2007; Lanza \& Satz, 1995).

Alguns estudos mostram, ainda, dificuldades éticas referentes a manejos técnicos em terapia de grupo, a formas de pagamento e a aumentos futuros em psicoterapia de grupo (Shapiro \& Ginzberg, 2006), ao encerramento de um grupo, à privacidade e ao relacionamento entre os pares (Mangione, Forti, \& lacuzzi, 2007), bem como àquelas advindas ao se trabalhar com a diversidade quando se coordena um grupo (Debiak, 2007).

Esses diversos impasses podem gerar entre coordenadores de grupo uma falta de acordo sobre respostas apropriadas em situações de dilema ético (McDivitt, 2001) e, consequentemente, a necessidade de que as organizações profissionais e programas de formação assumam o compromisso com uma prática grupal ética (Brabender, 2007).

Buscando orientar os profissionais na solução desses dilemas, algumas raras associações internacionais e suas divisões que congregam os profissionais que realizam práticas grupais têm buscado desenvolver normatizações éticas específicas para o trabalho realizado nesse contexto. Entre essas associações, podemos destacar: a American Group Psychotherapy Association (AGPA, Associação Americana de Psicoterapia de Grupo), a International Association for Group Psychotherapy and Group Processes (IAGP, Associação Internacional de Psicoterapia de Grupo e Processos Grupais) e a Association of Specialists in Group Work (ASGW, Associação de Especialistas em Trabalho de Grupo). No Brasil, contudo, ainda não dispomos de nenhuma associação que congregue a maioria dos profissionais que trabalham em grupos, não havendo, assim, nenhuma orientação ou diretriz específica ao trabalho realizado nesse contexto. 
Considerando o conjunto de desafios experimentados pelos coordenadores de grupo e a lacuna de orientações no contexto brasileiro, e buscando contribuir para a reflexão sobre o discurso ético nas práticas grupais, este artigo tem por objetivo compreender de que forma documentos de associações profissionais voltadas à prática grupal descrevem e propõem a ética em grupo.

Metodologicamente, será realizada uma análise crítica dos principais temas presentes em documentos internacionais. Trata-se de uma análise descritiva e comparativa sobre a visão de ética, de grupo e do papel do coordenador do grupo. Assim, analiticamente, não se adota uma perspectiva ética a priori, mas busca-se identificar aquela emergente dos documentos. A escolha por analisar os documentos dessas instituições se deve: a) à escassez desse tipo de documento; b) à abrangência dos documentos, que incluem práticas de psicoterapia de grupo e outras práticas de grupo; e c) a importância dessas associações no contexto internacional das práticas grupais.

Esperamos que esse estudo possa contribuir para a compreensão dos caminhos encontrados por tais associações para o enfrentamento dos impasses éticos e para a reflexão sobre suas potencialidades e desafios, permitindo, assim, a ampliação do imaginário ético nas práticas grupais e seu poder transformador.

\section{Documentos de orientação ética para a prática grupal}

Visando ao objetivo proposto, a análise busca contextualizar a produção de alguns documentos sobre ética apresentados por associações internacionais para então identificar a visão de ética e de grupo e o papel do coordenador do grupo por eles promovido. Os documentos a serem analisados são: as Diretrizes para a Melhor Prática da ASGW (Association for Specialists in Group Work Executive Board [ASGW], 2008), as Diretrizes Éticas da Associação Americana de Psicoterapia de Grupo e do Registro Nacional de Psicoterapeutas de Grupo Certificados (American Group Psychotherapy Association [AGPA], 2002), e as Diretrizes Éticas e
Padrões Profissionais para a Psicoterapia de Grupo (International Association for Group Psychotherapy and Group Processes [IAGP], 2009).

\section{Um panorama dos documentos: contexto, estrutura e objetivos}

Os três documentos analisados foram produzidos por associações americanas e internacionais da área de aconselhamento e psicoterapia de grupo que congregam diversos profissionais da área de Psicologia. Os documentos foram criados ou revistos na última década e apresentam diferentes níveis de complexidade. De forma geral, essas associações produziram documentos que constituem uma tentativa de uma descrição abrangente e totalizante sobre uma prática grupal ética que pode ser utilizada por profissionais que trabalham com grupos em diferentes áreas da atuação, apesar de apresentarem certo predomínio da saúde mental, especialmente, da clínica. Sinteticamente, pode-se ver (Tabela 1), as principais características de cada um desses documentos.

A análise das funções dessas diretrizes exige a compreensão dos tipos de documentos que elas constituem no campo da ética profissional. Nesse sentido, podemos falar de princípios, códigos, diretrizes éticas e guias de boas práticas. Os princípios éticos inspiram as ações humanas em diferentes dimensões e podem ser expressos, no meio profissional, por meio de diferentes documentos. Os códigos de ética para a atuação profissional são propostos por associações, exigem determinados comportamentos e requerem a adesão de seus membros. As diretrizes éticas também são desenvolvidas por associações e, apesar de não instruírem o manejo de situações específicas, potencialmente, oferecem parâmetros mais claros a serem considerados pelos profissionais. De forma semelhante, porém de forma claramente associada a procedimentos cotidianos, os guias de boas práticas descrevem ações que sustentam uma atuação profissional de alta qualidade (AGPA, 2007; Gilman, 2005).

Os três documentos analisados neste estudo se definem, a partir de seu título e conteúdo, como diretrizes éticas e guias de boas práticas. Seus obje- 
Tabela 1

Principais aspectos dos documentos sobre ética

\begin{tabular}{|c|c|c|c|}
\hline Aspectos & $\begin{array}{l}\text { Diretrizes para a melhor } \\
\quad \text { prática da ASGW }\end{array}$ & $\begin{array}{l}\text { Diretrizes éticas da AGPA e } \\
\qquad \text { NRCGP }\end{array}$ & $\begin{array}{c}\text { Diretrizes éticas e padrões } \\
\text { profissionais para a psicoterapia } \\
\text { de grupo }\end{array}$ \\
\hline Associação proponente & ASGW & AGPA & IAGP \\
\hline Ano de publicação & 2007 & 2002 & 2009 \\
\hline Profissionais-alvo & Conselheiros & Multidisciplinar & Multidisciplinar \\
\hline Estrutura formal & 3 seções / 22 itens / 23 subitens & 2 seções / 6 itens / 12 subitens & 9 itens / 62 subitens \\
\hline Tipos de grupo & $\begin{array}{l}\text { Psicoterapia, aconselhamento, } \\
\text { trabalho, psicoeducação }\end{array}$ & Psicoterapia de grupo & Psicoterapia de grupo \\
\hline $\begin{array}{l}\text { Dimensões da prática ética e } \\
\text { estrutura do documento }\end{array}$ & $\begin{array}{l}\text { (A) Planejamento (A1 a A9); } \\
\text { (B) Desempenho (B1 a B9), e } \\
\text { (C) Processamento dos grupos } \\
\text { (C1 a C4). }\end{array}$ & $\begin{array}{l}\text { Responsabilidade com o cliente: } \\
\text { (1) autonomia (1.1. a 1.3); } \\
\text { (2) privacidade (2.1 a 2.5); } \\
\text { (3) proteção contra prática incom- } \\
\text { petente (3.1 a 3.4). } \\
\text { Padrões profissionais (1 a 3) }\end{array}$ & $\begin{array}{l}\text { (1) Princípios gerais (1.1. a 1.7); } \\
\text { (2) Enquadre do tratamento } \\
\text { (2.1 a 2.8); } \\
\text { (3) Confidencialidade (3.1 a 3.9); } \\
\text { (4) Relações do tratamento } \\
\text { (4.1 a 4.5); } \\
\text { (5) Pesquisa (5.1 a 5.6); } \\
\text { (6) Educação (6.1 a 6.12); } \\
\text { (7) Educação continuada (7.1); } \\
\text { (8) Relação com colegas } \\
\text { (8.1 a 8.12); } \\
\text { (9) Questões sociais. }\end{array}$ \\
\hline
\end{tabular}

Nota: ASGW: Association of Specialists in Group Work; AGPA: American Group Psychotherapy Association; IAGP: International Association for Group Psychotherapy and Group Processes.

tivos são oferecer um "modelo para o comportamento ético do terapeuta de grupo" (AGPA, 2002) a fim de promover uma maior consciência profissional e uma prática de alto nível (IAPG, 2009) e apontar, de forma prática, suas responsabilidades no desenvolvimento do trabalho (ASGW, 2008). Enquanto o documento da AGPA se coloca como um conjunto de diretrizes, o da ASGW se define como um guia, e o da IAGP é uma combinação de ambos os formatos.

Apesar de, formalmente, serem de tipos semelhantes, eles possuem diferentes inserções institucionais, pois os documentos da AGPA e IAGP consistem de documentos primeiros, portanto, referência principal de seus associados. É importante observar que eles não estão referidos a um código de ética próprio da associação, sendo aos seus membros requerido que sigam o código de ética de sua filiação profissional original. Já o documento da ASGW é, institucionalmente, secundário, na forma de um guia de boas práticas, que busca "tornar 408 clara a aplicação do Código de Ética da American
Counseling Association (ACA, Associação Americana de Aconselhamento) ao campo do trabalho de grupo" (ASGW, 2008, p.111).

Em relação ao formato, observa-se que o documento da AGPA é muito breve, apontando de forma global alguns princípios e orientações éticos. Já os documentos da ASGW e IAPG são extensos e abarcam com maior detalhe tanto os princípios como os comportamentos esperados de seus associados. Assim, o documento da IAGP versa sobre aspectos relativos à pesquisa (item 5), ao relacionamento com outros profissionais (item 8) e à participação em debates públicos (item 9), explorados brevemente nos outros documentos. Da mesma forma, o documento da ASGW, estruturado a partir da prática de planejamento (seção A), desenvolvimento (seção B) e avaliação grupal (seção C), detalha orientações sobre o processo grupal não abarcadas nos outros documentos.

Vê-se, assim, que são documentos criados no contexto americano ou internacional, resultado de demandas próprias a esse contexto. Recente- 
mente criados ou revisados, o que aponta para a importância desse debate para a área, têm a forma de diretrizes ou guias de boas práticas, buscando criar parâmetros pragmáticos para as ações dos coordenadores de grupo. São, mais comumente, documentos secundários, submetidos a outros códigos de ética, e apresentam certo predomínio de preocupações e orientações típicas da área clínica.

A fim de compreender de que forma esses documentos contribuem para o debate sobre ética, explora-se, a seguir, a visão de ética e de boa prática sustentada por eles.

\section{Sobre a visão de ética e a boa prática}

Os três documentos, como referências éticas, estão sustentados por um conjunto de valores e obrigações morais. Por vezes, tal sustentação é explícita, por vez, é subentendida. Na tentativa de identificar os valores promovidos e sua tradução em práticas profissionais, analisam-se as diretrizes propostas nos três documentos.

Uma análise de seus preâmbulos, em que tipicamente se apresentam os princípios éticos de um código ou diretriz, pouco contribui para compreender a visão de ética por eles proposta. O documento da IAGP, apesar de apresentar uma seção de princípios gerais, apenas o situa em acordo com a Declaração dos Direitos Humanos das Nações Unidas, sem explorar os valores gerais do documento. Nesse preâmbulo, o documento proíbe a participação do terapeuta de grupo em ações de tortura, em tratamento em condições humilhantes, e a imposição de convicções pessoais aos pacientes. As Diretrizes da AGPA apresentam uma descrição sobre o processo de queixa ética e a suspensão da licença do terapeuta. Já o guia de boas práticas da ASGW, de forma mais clara, explicita a visão da ética "como parte integrante do trabalho de grupo e visualiza os que fazem esse trabalho de grupo como agentes éticos" (ASGW, 2008, p.111), trazendo uma dimensão processual e intrínseca ao fazer ético.

Segundo análise das diretrizes propriamente ditas, de forma semelhante, os três documentos abordam aspectos referentes a cuidados com o paciente, com o grupo e a formação do coordenador de grupo. Há uma ênfase em orientações sobre a autonomia do membro do grupo, a confidencialidade e a prática grupal competente. A preocupação subjacente a essa ênfase sugere que tais documentos se sustentem em princípios da bioética principialista (Pegoraro, 2008): autonomia, beneficência, não maleficência e justiça. Há alguns autores, tais como Knapp e VandeCreek (2006), que sugerem que, no campo da Psicologia, a ética principialista também deve considerar os imperativos de fidelidade e responsabilidade pública. Foge ao escopo deste estudo uma apresentação dos principais sistemas éticos. Para uma visão panorâmica sobre as éticas em Psicologia e no campo da saúde, sugere-se a leitura de Figueiredo (1995) e Pegoraro $(2002,2008)$. Analisemos, então, se esses princípios estão presentes naqueles três documentos e a maneira como eles estão.

A autonomia se refere à capacidade humana de se autolegislar, de agir com liberdade, de tomar decisões, de se impor limites e se afirmar como pessoa. Ela pode ser traduzida como a busca de envolver o paciente nas decisões sobre seu próprio tratamento. Nos documentos analisados, a autonomia está subjacente a diferentes diretrizes, sejam referentes ao processo de esclarecimento sobre o grupo e obtenção de consentimento informado, no atendimento (AGPA, 1.1; ASGW, A.6, A.7; IAGP 2, 2.3) ou na pesquisa envolvendo grupos (IAGP, 5.2); sejam relativas à participação do membro no grupo (de assistência e/ou pesquisa) apenas enquanto apropriado a suas necessidades (AGPA 1.2; IAGP 2.2, 5.2, 5.3).

Já a beneficência impõe que sempre se faça o bem, oferecendo, profissionalmente, o melhor tratamento. Nos três documentos, essa possibilidade envolve a avaliação quanto à formação profissional adequada para realizar as atividades tanto sob a forma de autoavaliação permanente das condições de atuação do líder/terapeuta (AGPA, 3.1; ASGW, A.3, B.1, C.1, C.2; IAGP, 2.7, 6.11), quanto de avaliação e requerimento institucional para a formação (ASGW A.1, A.2, B2, B.3; IAGP, 6, 6.1 a 6.5) ou supervisão e formação continuada (AGPA 
PP1, ASGW A.8, A.9, C.1; IAGP, 5, 7, 7.1). Ela também envolve uma análise da adequação do serviço às necessidades dos participantes, abrangendo, assim, uma avaliação das condições grupais e do plano de trabalho, e as necessidades dos participantes, desde suas especificidades pessoais até necessidades de encaminhamento ou coterapia (ASGW, B.3, B.4, B.7, B.8, B.9, C.1, C.3; IAGP, 2.5, $2.6,2.8,8.3,8.12$ ).

A não maleficência proíbe que se faça o mal. Nos documentos analisados, ela está presente em diretrizes que proíbem: a tortura e o atendimento em condições degradantes (IAGP, 1.2, 1.3), a realização de pesquisas com riscos de danos graves aos participantes (IAGP, 5.1) e a imposição de valores pessoais aos pacientes (IAGP 1.6). Ela também se traduz em diretrizes que problematizam relações múltiplas do líder/terapeuta com os membros de um grupo (IAGP, 4.3), seja sob a forma de proibição de relações sexuais (AGPA 3.3; IAGP, 4.1, 4.2, 6.8), negociações financeiras (AGPA, 3.2; IAGP, 4.5, 6.8), ou de obtenção de vantagens pessoais (AGPA 3.2, $I A G P$, 4). Na ASGW, pondera-se que as relações entre o líder e os membros podem variar conforme o tipo de grupo (ASGW, B.3).

A justiça está associada à distribuição igual de recursos, bem como o tratamento igual entre as pessoas. No que se refere ao primeiro entendimento de justiça, ela pode se referir a uma avaliação do contexto de realização dos grupos e das necessidades e recursos locais (ASGW A.3, A.4, A.5). Em relação ao segundo entendimento, ele se traduz, principalmente, na proibição de atitudes discriminatórias (AGPA, 1.3; IAGP, 1.6) e na promoção do respeito à diversidade na relação com os potenciais participantes do grupo (ASGW, B.8; IAGP 1.5).

O princípio da fidelidade está ligado à veracidade, à lealdade, ao cumprimento das obrigações e à manutenção das promessas. Ele se expressa, nos documentos analisados, em diretrizes e orientações relacionadas à manutenção da confidencialidade e seus limites, no trabalho em grupo (AGPA 2, 2.1 a 2.5; ASGW A.6, A.7; IAGP, 3, 3.1 a 3.9), na pesquisa em grupo (IAGP, 5.4, 5.5), ou na formação do líder/terapeuta (IAGP, 6.9).

Finalmente, o princípio da responsabilidade 410 pública se refere à responsabilidade para com a sociedade, sendo uma forma geral de beneficência. Ele pode ser encontrado na diretriz de contribuir com a comunidade em geral (IAGP, 9), bem como no cultivo da democracia e das liberdades sociais (IAGP, 1.1). De forma mais específica, ele se traduz na responsabilidade do coordenador de grupo com sua categoria profissional e campo de conhecimento por meio da participação no desenvolvimento do conhecimento sobre grupos (AGPA, PP.2; ASGW, C.3; IAGP, 5.6), da formação adequada de profissionais (IAGP, 6.7, 6.10), da relação zelosa entre líderes/terapeutas de grupo (IAGP, 8, 8.1 a 8.12) ou da colaboração com a organização a que está filiado ou com outras instituições (ASGW, C.4; IAGP, 8.8, 8.9, 8.10). Além disso, em uma perspectiva de controle, o princípio da responsabilidade pública sustenta a orientação de proteção do público de práticas incompetentes e informações errôneas (AGPA 3.2, 3.4; IAGP, 6.12, 8.1), e a necessidade de reportar as violações éticas cometidas por outrem (AGPA, 3.4, PP 3; IAGP, 8.5, 8.11) e de orientar o paciente/o público sobre como proceder com queixas éticas (IAGP, 8.6).

Como visto, em relação aos princípios, as diferenças existentes entre os três documentos se colocam de forma complementar, não havendo uma contradição clara entre eles. Além disso, podemos observar que os documentos sustentam ora uma ética obrigatória, ora uma ética de princípios (Pedersen, 1997), ou seja, há uma mistura de orientações que sugerem uma abordagem do controle e da prescrição, mesclada com outras orientações pautadas por uma abordagem positiva e inspiradora de padrões éticos. Trata-se, assim, de documentos formalmente híbridos que sustentam uma visão pragmática sobre a ética e a boa prática profissional.

Coerentemente com essa visão prática, considera-se que, apesar desses princípios poderem estar relacionados a diferentes sistemas éticos, sua presença, tal como sugerido anteriormente, dá-se a partir de uma influência da perspectiva principialista, muito comum no contexto contemporâneo na área de saúde (Pegoraro, 2008). Entende-se, assim, que os referidos documentos são marcados pela sua inserção sócio-histórica e sintonizados com outros documentos de diferentes categorias pro- 
fissionais preocupadas com questões éticas e qualidade dos serviços oferecidos. Eles funcionam, assim, como uma forma de aproximar, ainda que de forma indireta e apenas no modelo administrativo, as associações grupais que os propõem às tradicionais instituições da área de saúde.

\section{Sobre a especificidade da questão grupal: a visão de grupo e o papel do coordenador e participantes}

Apesar da semelhança nos princípios gerais que orientam os documentos analisados, no que se refere à especificidade da definição e ao cuidado ético na condução dos grupos, há uma grande diferença entre os documentos da AGPA e da IAGP em relação ao da ASGW. Essa diferença pode ser percebida na definição de grupo e no caráter das orientações éticas voltadas ao processo grupal.

No que se refere à visão de grupo, é curioso observar que nenhum dos documentos analisados define claramente o que é a prática grupal. Os documentos da IAGP e da AGPA se referem à psicoterapia de grupo sem caracterizá-la, e subentendendo-se, a maior parte do tempo, grupos de longo prazo ou de término indefinido. Diferentemente, no guia da ASGW, são apontados quatro tipos de prática/contexto grupal: psicoterapia, aconselhamento, trabalho e psicoeducação.

Em relação ao caráter das orientações éticas, nas recomendações da AGPA e da IAGP, poucas orientações são dirigidas e tentam responder às questões cotidianas referentes ao processo grupal propriamente dito, sendo a maior parte referente a posturas éticas a serem empregadas pelos profissionais coordenadores de grupo em diferentes contextos de sua atuação. A especificidade grupal aparece associada de forma mais clara à questão da confidencialidade e da privacidade, indicando a importância de se considerar que todos os participantes são responsáveis pela manutenção da confidencialidade e privacidade (AGPA, 2.1; IAGP, 3.1, $3.7,3.8)$. Contudo, a própria definição da confidencialidade, apesar de importante em muitos contextos, especialmente clínicos, pode ter um caráter secundário a depender do tipo de grupo que se desenvolva e os objetivos que se busque. O que se observa é que há nesses documentos o predomínio de uma visão tradicional de grupo, pautada por uma perspectiva clínica, de consultório, privada, centrada no profissional, fortemente marcada pelos mesmos orientadores da psicoterapia individual (Zimmerman \& Osório, 1997).

No documento da ASGW, a consideração do grupo é mais abrangente, incorporando desde uma avaliação ecológica do grupo (ASGW, A3), relativa aos seus recursos e à inserção do grupo em determinado meio social, passando por orientações sobre o desenvolvimento do grupo (tipo, participação dos membros, estilo de liderança) (ASGW, A4) e adaptação do grupo (estágio do grupo/tipo de grupo) (ASGW, B3), até a colaboração entre os membros (ASGW, B6), pautada por uma sensibilidade cultural quanto à forma de interação dos participantes (ASGW, B8) e uma atenção à dinâmica dos membros (ASGW, C2). Há, assim, uma visão de grupo mais ampla, contextualizada, realizada em contextos variados, com diferentes objetivos, preocupada com o processo grupal, com a interação entre os membros e com uma sensibilidade cultural. Preocupações tradicionais com a necessidade de confidencialidade e a evitação de relações múltiplas entre coordenador de grupo e participante, apesar de presentes, são redimensionadas e não apenas exigidas ou proibidas. Trata-se de uma visão sobre a prática grupal, potencialmente, mais útil a uma maior variedade de profissionais que trabalham com grupos.

Essas diferenças entre os vários documentos podem ser entendidas no contexto das associações que os produziram. Temos associações voltadas, de um lado, predominantemente, à psicoterapia de grupo (AGPA e IAGP) e, de outro, ao trabalho em grupo (ASGW) de formas mais variadas. A heterogeneidade das práticas desse último convida a um maior detalhamento das considerações sobre o grupo, o coordenador e os participantes.

Apesar de não se definir o grupo, define-se quem é o profissional. Nos documentos da AGPA e na IAGP, o profissional que trabalha com grupo é sempre nomeado como psicoterapeuta ou terapeuta de grupo. Seu papel é descrito de forma a 
apagar sua especificidade grupal e enfatizar sua semelhança com qualquer outro terapeuta. No documento da ASGW, o profissional é nomeado, predominantemente, como líder do grupo. Seu papel, como vimos, envolve diferentes ações referentes ao planejamento, desenvolvimento e processamento do grupo, mais próximas ao fazer grupal cotidiano. Apesar disso, há várias responsabilidades do líder do grupo comuns a outros profissionais da área de Psicologia.

Aos participantes, por sua vez, não é reservado um papel significativo no enfrentamento das questões éticas. Novamente, com exceção das orientações referentes à preservação da confidencialidade (prevista nos três documentos) e ao respeito aos membros do grupo como parceiros iguais (presente no guia de boas práticas), não é atribuída nenhuma outra responsabilidade aos participantes na construção e na manutenção das condições éticas no grupo. Assim, apesar de vários autores no campo do trabalho em grupos (Guanaes, 2006; Rogers, 2002) ressaltarem o papel de todos os envolvidos, terapeutas e participantes, no processo grupal, com responsabilidade nos cuidados uns com os outros, nos documentos analisados, essa ética é pouco explorada. A ética é reduzida ao papel profissional do líder terapeuta.

\section{Sobre documentos de ética para a prática grupal: para além das regras}

A análise realizada permitiu identificarmos o contexto e a forma de diretrizes e guia de boas práticas dos documentos da AGPA, IAGP, e ASGW. Foi possível ainda reconhecer a predominância do caráter institucionalmente secundário dos documentos analisados; a hegemonia de uma ética principialista promotora da autonomia, beneficência, não maleficência, justiça, fidelidade e responsabilidade pública; um silenciamento sobre as definições de grupo e o papel do participante nas questões éticas; e a ênfase de diretrizes voltadas ao papel profissional do terapeuta, sendo pouco específicas do trabalho grupal, especialmente nos documentos

412 da AGPA e IAGP.
Tais entendimentos apontam para uma descrição profissionalizante sobre a ética (Rasera \& Carrijo, 2011) centrada no papel do coordenador do grupo, e relegando a segundo plano uma perspectiva ética processual e dialogicamente negociada, relativa ao desenvolvimento co-construído do grupo entre todos os envolvidos. Mais inquietante ainda é o foco das orientações ao líder/terapeuta em ações que não sejam específicas ao contexto grupal e que consistam em uma sobreposição e repetição de diretrizes já presentes nos códigos de éticas dos profissionais da área de Psicologia. Essa descrição profissionalizante associada ao silenciamento sobre as questões grupais oferece poucas contribuições ao terapeuta em sua prática cotidiana. Assim, se se tomar as principais problemáticas éticas vividas pelos profissionais que trabalham com grupos, tal como apresentado na literatura, as diretrizes e orientações, apesar de estabelecerem parâmetros para uma prática ética, pouco facilitam seu enfrentamento.

Dessa forma, esses documentos, para além de descreverem os aspectos éticos do trabalho grupal, contribuem para a criação de um arcabouço administrativo, burocrático, quase jurídico para a sustentação do 'profissional' do trabalho grupal, especialmente da terapia de grupo, como se estivessem estabelecendo uma norma de uma especialidade profissional (Pryzwansky \& Wendt, 1999). Eles pouco abordam a ética específica do grupo. Comparados com outros códigos da área de Psicologia, eles têm preocupações e orientações semelhantes referentes aos padrões éticos de comportamento profissional.

Essa aparente contradição pode ser explicada a partir da compreensão de que, da forma como se apresentam, tais documentos contribuem com a institucionalização da profissão, formalizam seus padrões e a valorizam frente à comunidade de profissionais e consumidores/usuários. Nesse sentido, Gilman (2005) aponta que um documento profissional de ética pode indicar tanto os comportamentos adequados e inadequados como a visão sobre o que é a profissão. Ele estabelece as transgressões, mas também os princípios que informam as ações. Ele define não apenas o modo de atuação profissional, mas como a profissão quer ser vista. 
Esses documentos, portanto, dão o contexto no qual a profissão se desenvolve, e são fundamentais no processo de sua afirmação.

Ao mesmo tempo, entendidos dessa maneira, os documentos analisados podem ser uma forma de responder a uma pressão social por responsabilização pela prática profissional, representada, em um contexto internacional, pelas agências financiadoras dos serviços realizados por terapeutas e líderes de grupo. Essa tendência tem crescido nos últimos anos, levando a AGPA a criar um documento amplo de diretrizes gerais para a prática profissional em 2009 (AGPA, 2007). A produção desses documentos se insere, assim, no contexto da competição mercadológica (Pryzwansky \& Wendt, 1999), contribuindo para uma visão positiva dos trabalhos de grupo, pautada pela imagem de instâncias organizadoras e reguladoras que promovem serviços com elevados padrões de qualidade.

Os documentos propostos pelas organizações lembram que os desafios éticos estão situados no contexto da responsabilidade profissional, contribuindo para uma visão institucional da ética. Se eles, por um lado, não permitem soluções práticas para o enfrentamento dos desafios cotidianos, por outro, chamam nossa atenção para o contexto social que produz, a partir do esforço em se definir como profissão, determinadas preocupações e, consequentemente, como resposta, orientações e diretrizes que contemplam certos aspectos da experiência grupal.

Acredita-se que, para além da afirmação de uma especialidade profissional, as normatizações éticas ofereçam um panorama moral para as decisões técnicas, as quais devem ser complementadas por um processo contínuo de formação e pelo desenvolvimento de modelos de decisão ética. Assim, a partir dessas orientações e habilidades, o líder terapeuta poderá, em conjunto com os participantes e de forma situada, melhor definir ações que promovam os princípios pretendidos em um processo de responsabilidade compartilhada (McNamee \& Gergen, 1999).

A análise realizada não buscou, portanto, 0 código, diretriz ou guia perfeito. Ela permitiu iden- tificar a visão de ética e de grupo e o papel do coordenador e dos participantes, possibilitando compreender o discurso ético proposto pelas organizações e reconhecer alguns sentidos disponíveis para aqueles que trabalham com grupos significarem sua prática e seus problemas.

\section{Referências}

American Group Psychotherapy Association. (2002). Guidelines for ethics. Retrieved December 1, 2011, from http://www.groupsinc.org/group/ethicalguide. html

American Group Psychotherapy Association. (2007). Practice guidelines for group psychotherapy. Retrieved December 1, 2011, from http://www.agpa.org/ guidelines/index.html

Association for Specialists in Group Work Executive Board. (2008). Association for specialists in group work: Best practice guidelines revisions 2007. Journal for Specialist in Group Work, 33(2), 111-117.

Brabender, V. (2007) The ethical group psychotherapist: A Coda. International Journal of Group Psychotherapy, 57(1), 41-47.

Conselho Federal de Psicologia. (2005). Código de Ética Profissional do Psicólogo. Brasília: CFP.

Conselho Regional de Psicologia de São Paulo. (2005). Novo código para novos tempos. Jornal de Psicologia, 145, 6-7.

Debiak, D. (2007). Attending to diversity in group psychotherapy: An ethical imperative. International Journal of Group Psychotherapy, 57(1), 1-12.

Figueiredo, L. C. M. (1995). Revisitando as psicologias. São Paulo: Vozes.

Gilman, S. C. (2005). Ethics codes and codes of conduct as tools for promoting an ethical and professional public service: Comparative successes and lessons. Washington: World Bank. Retrieved September 11, 2011, from http://www.oecd.org/dataoecd/17/33/ 35521418.pdf

Guanaes, C. (2006). A construção da mudança em terapia de grupo. São Paulo: Vetor.

Hough, G. (1992). When confidentiality mandates a secret be kept: A case report. International Journal of Group Psychotherapy, 42(1), 105-115.

International Association for Group Psychotherapy and Group Processes. (2009). Ethical guidelines and professional standards for group psychotherapy. Retrieved December 1, 2011, from http://www.iagp. com/about/ethicalguidelines.htm

Knapp, S., \& VandeCreek, L. D. (2006). Practical ethics for psychologists: A positive approach. Washington: American Psychological Association. 
Lanza, M. L., \& Staz, H. (1995). Researcher and clinician: Role conflict and resolution. Group, 19(2), 120-126.

Mackenzie, K. R. (1999). Professional ethics and the group psychotherapist. Group Solution. Retrieved August 12, 2009, from http://www.groupsinc.org/pubs/GS_ 0499.html

Mangione, L., Forti, R., \& lacuzzi, C., M. (2007). Ethics and endings in group psychotherapy: Saying goodbye and saying it well. International Journal of Group Psychotherapy, 57(1), 25-40.

McDivitt, K. L. (2001). Ethics in group work with children and adolescents. Dissertation Abstracts International: A, The Humanities and Social Sciences, 61(12A), 4673.

McNamee, S., \& Gergen, K. J. (1999). Relational responsibility: Resources for sustainable dialogue. Thousand Oaks: Sage.

Merta, R. J., \& Sisson, J. A. (1991). The experiencial group: An ethical and professional dilemma. Journal for Specialists in Group Work, 16(4), 236-245.

Motta, K. A. M. B., Nunes, F. C., Munari, D. B., \& Medeiros, M. (2007). O grupo como instrumento de construção do conhecimento: aspectos éticos. Revista da SPAGESP, 8(1), 4-13.

Pedersen, P. (1997). The cultural context of the American counseling association code of ethics. Journal of Counseling and Development, 76(1), 23-28

Pegoraro, O. (2002). Ética e bioética: da subsistência à existência. Petrópolis: Vozes.

Pegoraro, O. (2008). Ética dos maiores mestres através da história. Rio de Janeiro: Vozes.
Pepper, R. S. (2002). Emotional incest in group psychotherapy. International Journal of Group Psychotherapy, 52(2), 285-294.

Pepper, R. S. (2004). Confidentiality and dual relationships in group psychotherapy. International Journal of Group Psychotherapy, 54(1), 103-114.

Pryzwansky, W. B., \& Wendt, R. N. (1999). Professional and ethical issues in psychology. New York: W. W. Norton.

Rasera, E. F., \& Carrijo, R. S. (2011). O que você faria numa situação dessa? Narrativas sobre ética e grupos. In E. F. Rasera, M. M. Castro, \& C. J. Van Stralen (Org.), Psicologia social: ética, participação política e inclusão social (pp.155-174). Curitiba: CRV.

Roback, H. B., Ochoa, E., Bloch, F., \& Purdon, S. (1992). Guarding confidentiality in clinical groups: The therapist's dilemma. International Journal of Group Psychotherapy, 42(1), 81-103.

Rogers, C. R. (2002). Grupos de encontro. São Paulo: Martins Fontes.

Shapiro, E. L., \& Ginzberg, R. (2006). Buried treasure: Money, ethics and countertransference in group therapy. International Journal of Group Psychotherapy, 56(4), 477-494.

Zimmerman, D., \& Osório, L. C. (1997). Como trabalhamos com grupos. Porto Alegre: Artes Médicas.

Recebido em: 2/2/2012

Versão final em: 10/7/2012

Aprovado em: 2/8/2012 